\title{
Large scale structure and the generalized Chaplygin gas as dark energy
}

\author{
Tuomas Multamäki ${ }^{1}$, Marc Manera ${ }^{2}$, Enrique Gaztañaga ${ }^{2}$ \\ ${ }^{1}$ Departament E.C.M. and C.E.R., Universitat de Barcelona, Diagonal 647, 08028 Barcelona, Spain and \\ 2 Insitut d'Estudis Espacials de Catalunya, IEEC/CSIC, Gran Capitán 2-4, 08034 Barcelona, Spain
}

(Dated:)

\begin{abstract}
The growth of large scale structure is studied in a universe containing both cold dark matter $(\mathrm{CDM})$ and generalized Chaplygin gas $(\mathrm{GCg}) . \mathrm{GCg}$ is assumed to contribute only to the background evolution of the universe while the CDM component collapses and forms structures. We present some new analytical as well as numerical results for linear and non-linear growth in such model. The model passes the standard cosmological distance test without the need of a cosmological constant (LCDM). But we find that the scenario is severely constrained by current observations of large scale structure. Any small deviations of the GCg parameters away from the standard Lambda dominated cosmology (LCDM) produces substantial suppression for the growth of structures.
\end{abstract}

\section{INTRODUCTION}

The current cosmological concordance model that is well supported by the latest microwave background and supernova type Ia observations, includes both a large repulsive cosmological term and a cold dark matter (CDM) component. The nature and origin of these components is yet unknown, leaving room for novel theoretical explanations and new cosmological scenarios. In the standard cosmological scenario the cosmological term, $\Lambda$, is a true constant but, as is already suggested by the plethora of papers devoted to the subject, this need not to be the case. In addition, the difficulty of explaining the existence and value of the cosmological constant from fundamental physics, leaves plenty of room and motivation for phenomenological proposals.

Instead of having a universe with a non-zero $\Lambda$, one can study cosmological models that, within the observational boundaries, imitate the evolution of a universe with a cosmological constant and cold dark matter, the $\Lambda$ CDM model. Such models can have be the result of a modified Friedmann equation or contain a new type of matter with positive energy density and negative pressure. Among these models are the large class of quintessence models (see e.g. [1]), the brane inspired models [2], Cardassian models [3] and recently the Chaplygin [41] gas models [4].

The General Chaplygin gas (GCg) models are characterized by a perfect fluid with a non-standard equation of state

$$
p_{G}=-\frac{\tilde{A}}{\rho_{G}^{\alpha}}
$$

where $\tilde{A}$ is a positive (dimensionfull) constant. It is useful to define a dimensionless constant, $A$ (also often denoted as $A_{s}$ ), by $A \equiv \rho_{G, 0}^{-(1+\alpha)} \tilde{A}$, where $\rho_{G, 0}$ is the present day density of the CGg.

The original Chaplygin gas model [4] corresponds to the choice $\alpha=1$. Such an equation of state can be the result of a scalar field with a non-standard kinetic term, e.g. the string theory motivated tachyon field [5, [6]. The generalization of the original Chaplygin gas, i.e. to cases where $\alpha \neq 1$, was done in [7]. Since the original Chaplygin gas scenario is reached as a special case of the general Chaplygin gas, we will concentrate on the latter.

The cosmological behavior of the GCg is between a dark matter and a cosmological constant. At large densities, it behaves as pressureless dust where as at small densities, i.e. presently, it acts as a cosmological constant. As such, one can hope to have a unified dark matter candidate, i.e. one that can play both the role of cold dark matter and of the cosmological constant [4, 8]. Taking a more conservative view, one can also consider a universe with both significant CDM and GCg components. The different cosmological models that include a (generalized) Chaplygin gas component can then be divided into two classes: models with and without a significant CDM component. Models that do not include a specific CDM component are often called unified dark matter (CDM) models.

The unified dark matter models with a (generalized) Chaplygin gas have been studied in various works in view of cosmological observations. Cosmological perturbations of the GCg-fluid have been considered in [7], [9]-[16]. In 12] it was claimed that the matter power spectrum strongly constrains the parameter space of unified dark matter models, essentially ruling them out. This result does not include the effect of the baryons, however, and is hence questionable 13. Also, as it was pointed out in [15, 17], the restriction due to the matter power spectrum may be avoided by e.g. allowing for entropy perturbations. To complicate the matter further, in 16] it was shown that one must be careful in using linear theory to study the perturbations as linear theory may break down sooner than expected.

Other observational measures of universes with a GCg-component include the SNIa constraints 8, 14, 18, 19, 20, 21, 22], constraints coming from the CMB [7, 14, 23, 25, 26, 27] and other astrophysical data [17, 24, 30]. 
A significant fraction of the work so far has concentrated on the unified dark matter proposal. In this letter we concentrate on the more general case where there is in addition to the GCg-component, a significant cold dark matter fraction. The GCg plays the role of dark energy, from this point of view this model can be seen as a quintessence model with a dynamical equation of state. GCg as dark energy has been studied in recent works 14, 18, 19, 21, 26, 30]. Interestingly, in 18, 19] it was found by using SNIa data that a universe dominated with a Chaplygin gas was favored over a mixed model. On the other hand, in [14] it was concluded that a $\Lambda$ CDM model is preferred. Also in [26], using WMAP data it was showed that a CDM model is disfavored and a mixed model, with a $\Lambda$ CDM -like behavior, was preferred. The current bounds on the parameters are varied, depending on the type observational data. Using globular clusters to determine the age of the universe, in 30 it was found that $\Omega_{M} \geq 0.2, A \geq 0.96(\alpha=1)$, where as for the lensing statistics the bounds are somewhat looser $\Omega_{M} \leq 0.45, A_{\geq} 0.72$. Using the WMAP data, in [26] the parameter bounds were found to be $A \geq 0.8,0 \leq \alpha \leq 0.2(95 \% C L)$ and the original Chaplygin gas is ruled out. In [21], X-rays from galaxy clusters, HST data and SNIa data were used to obtain $A \geq 0.84,0.273 \leq \Omega_{M} \leq 0.329(95 \% C L)$. Using only SNIa data, it was found in [18] for the original Cg-model that $A=0.93_{-0.20}^{+0.07}, 0 \leq \Omega_{M} \leq 0.35$.

In this work we study the mixed scenario, with CDM and GCg, from the point of view of large structure growth. We take a quintessence-like approach to the Chaplygin gas, i.e. only the CDM component is assumed to collapse and form structures. The Chaplygin gas acts only as dark energy and is visible, from the point of structure growth, only through the Friedmann equation. This is to be contrasted with the unified dark matter scenario where the $\mathrm{Cg}$ is also responsible for the growth of structures. The scenario considered here may be seen e.g. as a universe dominated by a scalar field with a non-standard kinetic term arising from string theory. Similar considerations for quintessence scenarios have been presented in [31], where large scale structure is used to test different quintessence models.

By studying large scale structure growth from the point of view of gravitational collapse we probe the significance of the GCg dark energy component throughout the epoch during which large scale structures grow. The linear and non-linear growth can be related to observations and hence lead to observational constraints like galaxy number counts (see 32]). Our approach is similar to [32, 33], where other non-standard cosmologies have been considered (also see 35] for related discussions). In Section 2 we review and study properties of GCg cosmology. The spherical collapse formalism is reviewed in Section 3 and applied to the GCg case, producing some analytical results. Numerical work is performed in Section 4. Discussion and conclusion is presented in Section 5.

\section{GENERALIZED CHAPLYGIN GAS COSMOLOGY}

Assuming in general that we have both a cold dark matter component and a GCg component, the Friedmann equation is

$$
H^{2} \equiv\left(\frac{\dot{a}}{a}\right)^{2}=\kappa^{2}\left(\rho_{M}+\rho_{G}\right)
$$

where $\kappa^{2}=8 \pi G / 3, \rho_{M}$ is the energy density of cold dark matter and $\rho_{G}$ of the generalized Chaplygin gas. Note that there is no explicit cosmological constant and that we choose work in a flat universe.

The continuity equation of the different components are

$$
\begin{array}{r}
\dot{\rho}_{M}+3 H\left(\rho_{M}+p_{M}\right)=0 \\
\dot{\rho}_{G}+3 H\left(\rho_{G}+p_{G}\right)=0,
\end{array}
$$

i.e. the two fluids interact only gravitationally. Using Eq. (11) and the fact that in a matter dominated universe $p_{M}=0$, we get two equations that can be solved straightforwardly:

$$
\begin{aligned}
& \rho_{M}=\frac{\rho_{M, 0}}{a^{3}} \\
& \rho_{G}=\rho_{G, 0}\left(A+(1-A) a^{-3(1+\alpha)}\right)^{\frac{1}{1+\alpha}},
\end{aligned}
$$

where the index 0 refers to present day values $\left(a_{0}=1\right)$ and $A\left(\equiv \rho_{G, 0}^{-(1+\alpha)} \tilde{A}\right)$ is a dimensionless constant, $0 \leq A \leq 1$. From Eq. (3), we see that at early times, the GCg behaves as matter, $\rho_{G} \sim a^{-3}$, whereas at late times it imitates a cosmological constant, $\rho_{G} \approx$ const.

Using the expressions (3) and the Friedmann equation, Eq. (2), one can look for solutions of $a(t)$ or $\rho(t)$. In the general case no analytical solution is found. As a special case, one sees that in a universe with only Chaplygin gas, i.e. $\rho_{M}=0$, the Friedmann equation is integrable and the solution is (up to a constant):

$$
t=\frac{2}{3}\left(\frac{1+\frac{A}{1-A} a^{3(1+\alpha)}}{A+(1-A) a^{3(1+\alpha)}}\right)^{\frac{1}{2(1+\alpha)}}{ }_{2} F_{1}\left(\frac{1}{2(1+\alpha)}, \frac{1}{2(1+\alpha)}, 1+\frac{1}{2(1+\alpha)}, \frac{A}{A-1} a^{3(1+\alpha)}\right) .
$$


In the same special case, one can also solve for the energy density (again, up to a constant):

$$
t=\frac{2}{3} \frac{1}{A \kappa(1+2 \alpha)} \frac{\rho^{\frac{1}{2}+\alpha}}{\rho_{0}^{1+\alpha}}{ }_{2} F_{1}\left(\frac{1+2 \alpha}{2(1+\alpha)}, 1, \frac{3+4 \alpha}{2(1+\alpha)}, \frac{\rho^{1+\alpha}}{A \rho_{0}^{1+\alpha}}\right)
$$

Using the solutions (3), we rewrite the Friedmann equation in terms of redshift as

$$
H^{2}=H_{0}^{2}\left(\Omega_{M}(1+z)^{3}+\Omega_{G}\left(A+(1-A)(1+z)^{3(1+\alpha)}\right)^{\frac{1}{1+\alpha}}\right),
$$

where we have defined $\Omega_{M}=\kappa^{2} \rho_{M, 0} / H_{0}^{2}$ and $\Omega_{G}=\kappa^{2} \rho_{G, 0} / H_{0}^{2}$. For $\alpha=0$ we see that Eq. (6) reduces to the $\Lambda$ CDM -model with $\Omega_{m}=\Omega_{M}+\Omega_{G}(1-A)$ and $\Omega_{\Lambda}=\Omega_{G} A$. Note that in a flat universe, our definitions imply that $\Omega_{M}+\Omega_{G}=1$.

As a first test, we should compare distances in the GCg cosmology with the standard $\Lambda$ CDM case. The luminosity distance $d_{L}$ is obtained from the (line-of-sight) comoving coordinate distance: $r(z)=\int d z^{\prime} / H\left(z^{\prime}\right)$. Fig. 1 shows predictions for different values of the model parameters for the corresponding apparent magnitude as compared to the SNIa results [28, 29]. It is apparent that current observations do not discriminate much between the different cosmological models. In other words: the GCg cosmologies seem to pass the standard cosmological distance test. It is then interesting to explore whether they also pass the observational constraints on the growth of linear and non-linear structures.

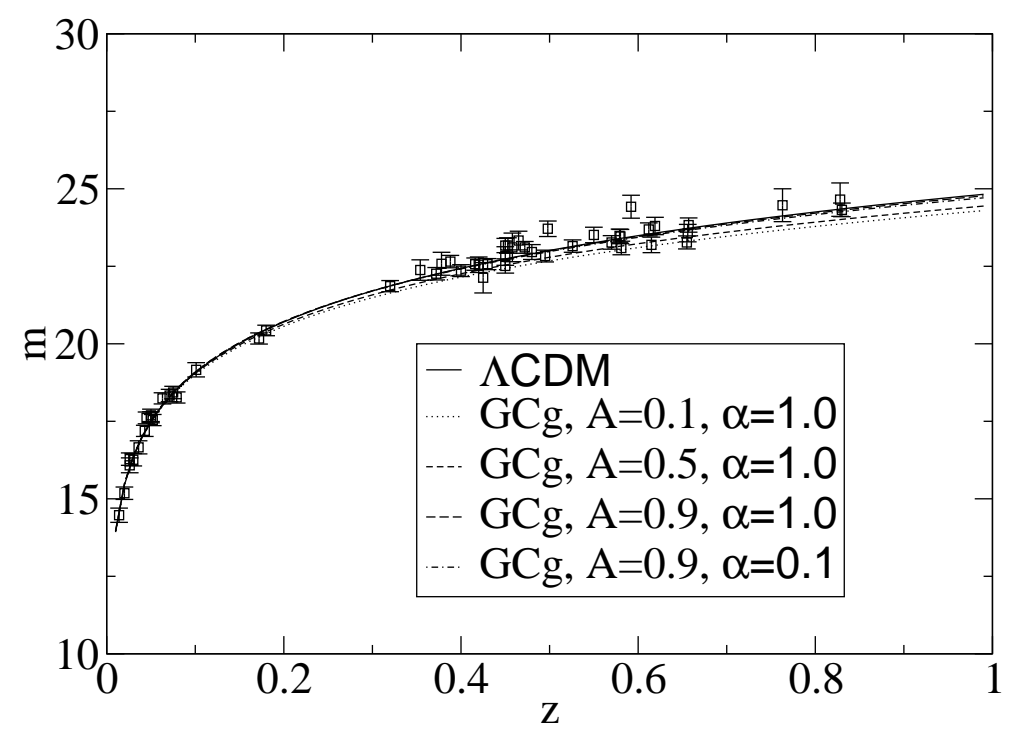

FIG. 1: Apparent magnitudes for SNIa for different cosmologies $\left(\Omega_{M}=0.3, \Omega_{G}=0.7\right)$.

\section{GRAVITATIONAL COLLAPSE}

The gravitational evolution of an over-dense region is dependent on the background evolution. A rapidly expanding background will obviously slow down the collapse of region compared to a static background. The details of the collapse are obviously complicated in a general case so here we choose work in a shear-free approximation and consider spherically symmetric configurations, i.e. we use the spherical collapse model to study the growth of large scale structure.

To study the dynamics of gravitational collapse, it is useful to define local density contrast as

$$
\delta=\frac{\rho}{\bar{\rho}}-1
$$


where $\bar{\rho}$ is the background energy density. Within the spherical collapse approximation one can show that in a matter dominated universe (i.e. the collapsing matter has no pressure) [32, 33.

$$
\frac{d^{2} \delta}{d \eta^{2}}+\left(2+\frac{\dot{\bar{H}}}{\bar{H}^{2}}\right) \frac{d \delta}{d \eta}-\frac{4}{3} \frac{1}{1+\delta}\left(\frac{d \delta}{d \tau}\right)^{2}=-3 \frac{1+\delta}{\bar{H}^{2}}\left(\left(\dot{H}+H^{2}\right)-\left(\dot{\bar{H}}+\bar{H}^{2}\right)\right)
$$

where $\eta=\ln (a), \bar{H}=\bar{H}(\bar{\rho})$ is the background Hubble rate and $H=H(\delta, \bar{\rho})$ is the local Hubble rate.

Working within the framework of small perturbations we expand $\delta$ as

$$
\delta=\sum_{i=1}^{\infty} \delta_{i}=\sum_{i=1}^{\infty} \frac{D_{i}(\eta)}{i !} \delta_{0}^{i}
$$

where $\delta_{0}$ is the small perturbation. Similarly, we can also expand the $\dot{H}+H^{2}$-term in terms of $\delta$ and then the whole RHS of Eq. (8) as

$$
3 \frac{1+\delta}{\bar{H}^{2}}\left(\left(\dot{H}+H^{2}\right)-\left(\dot{\bar{H}}+\bar{H}^{2}\right)\right) \equiv 3(1+\delta) \sum_{n=1} c_{n} \delta^{n}
$$

Using this and expanding the perturbation according to Eq. (9) we find the linear equation,

$$
D_{1}^{\prime \prime}+\left(2+\frac{\dot{\bar{H}}}{\bar{H}^{2}}\right) D_{1}^{\prime}+3 c_{1} D_{1}=0
$$

the second order equation,

$$
D_{2}^{\prime \prime}+\left(2+\frac{\dot{\bar{H}}}{\bar{H}^{2}}\right) D_{2}^{\prime}-\frac{8}{3}\left(D_{1}^{\prime}\right)^{2}+3 c_{1} D_{2}+6\left(c_{1}+c_{2}\right) D_{1}^{2}=0,
$$

and one can go on to arbitrary order by using the solutions to the lower order equations.

The second order equation can be related to the skewness of the density field at large scales (see [34] for more details). For Gaussian initial conditions,

$$
S_{3}=3 \frac{D_{2}}{D_{1}^{2}}
$$

In an Einstein-deSitter universe,

$$
\begin{aligned}
c_{1} & =-\frac{1}{2} \\
c_{i} & =0, i=2,3, \ldots \\
2+\frac{\dot{\bar{H}}}{\bar{H}^{2}} & =\frac{1}{2}
\end{aligned}
$$

and the well known solution to the linear equation (11) is

$$
D_{1}=B_{1} e^{\eta}+B_{2} e^{-\frac{3}{2} \eta}=B_{1} a+B_{2} a^{-\frac{3}{2}} .
$$

The skewness can also be calculated analytically (see 34]):

$$
S_{3}^{E d S}=\frac{34}{7} \approx 4.86
$$

\section{A. Gravitational collapse in GCg}

The $\dot{H}+H^{2}$-term in the GCg model is

$$
\dot{H}+H^{2}=-\frac{1}{2} \kappa^{2}\left(\rho_{M}+\rho_{G}\left(1-3 \frac{\tilde{A}}{\rho_{G}^{1+\alpha}}\right)\right)
$$


The gravitational dynamics in the GCg model obviously depend on the type of scenario i.e. whether we have a separate dark matter component or not and which components are fluctuating to form structures.

Assuming that GCg only affects large scale structure growth through its effect on the background evolution, $i . e$. only the fluctuations of the CDM component are responsible for large scale structure, we can write $\rho_{M}=\bar{\rho}_{M}(1+\delta)$, $\rho_{G}=\bar{\rho}_{G}$. Expanding according to (10) we find that

$$
\begin{aligned}
c_{1} & =-\frac{1}{2} \frac{\bar{\rho}_{M}}{\bar{\rho}_{M}+\bar{\rho}_{G}}=-\frac{1}{2} \frac{\Omega_{M}}{\Omega_{M}+\Omega_{G}\left[1+A\left(a^{3(1+\alpha)}-1\right)\right]^{\frac{1}{1+\alpha}}} \\
c_{2} & =0 \\
2+\frac{\dot{\bar{H}}}{\bar{H}^{2}} & =\frac{1}{2}\left(1+3 \frac{\tilde{A}}{\bar{\rho}_{G}^{\alpha}\left(\bar{\rho}_{M}+\bar{\rho}_{G}\right)}\right)=\frac{1}{2}\left(1+\frac{3 A \Omega_{G}\left[A+(1-A) a^{-3(1+\alpha)}\right]^{-\frac{\alpha}{1+\alpha}}}{\Omega_{M} a^{-3}+\Omega_{G}\left[A+(1-A) a^{-3(1+\alpha)}\right]^{\frac{1}{1+\alpha}}}\right)
\end{aligned}
$$

From Eqs (20) the small a limit, i.e. the early time limit, is easily read:

$$
\begin{aligned}
c_{1} & =-\frac{1}{2} \frac{\Omega_{M}}{\Omega_{M}+\Omega_{G}(1-A)^{\frac{1}{1+\alpha}}} \\
2+\frac{\dot{\bar{H}}}{\bar{H}^{2}} & =\frac{1}{2} .
\end{aligned}
$$

Comparing this to the linear equation in a $\Lambda \mathrm{CDM}$-universe,

$$
D_{1}^{\prime \prime}+\frac{1}{2} \frac{\Omega_{M}+4 a^{3} \Omega_{\Lambda}}{\Omega_{M}+a^{3} \Omega_{\Lambda}} D_{1}^{\prime}-\frac{3}{2} \frac{\Omega_{M}}{\Omega_{M}+a^{3} \Omega_{\Lambda}}=0,
$$

we see that a GCg-universe behaves fundamentally differently from the $\Lambda$ CDM -universe: at early times, $a \ll 1$, $\Lambda \mathrm{CDM}$-universe reduces to the EdS-case, with solution given by Eq. (17), where as the GCg-universe does not. Accordingly, the solution to the linear equation in the GCg-case (in the small $a$ limit) is

$$
D_{1}=B_{1} e^{\frac{1}{4}(\sqrt{1+24 \xi}-1) \eta}+B_{2} e^{\frac{1}{4}(-\sqrt{1+24 \xi}-1) \eta}
$$

where

$$
\xi=\frac{\Omega_{M}}{\Omega_{M}+\Omega_{G}(1-A)^{\frac{1}{1+\alpha}}} .
$$

Hence, EdS-case is recovered in the limits $\Omega_{G}=0$ and $A=1$. Compared to the EdS-case (and $\Lambda$ CDM -universe), fluctuations therefore start to grow more slowly in a universe where GCg plays a role. Defining $\epsilon=(1-A)^{1 /(1+\alpha)} \Omega_{G} / \Omega_{M}$, we see that the growing mode goes as (when $\epsilon \ll 1$ )

$$
D_{1} \sim a^{1-\frac{3}{5} \epsilon}
$$

On the other hand, in the large $a$ limit,

$$
D_{1}^{\prime \prime}+\frac{1}{2} \frac{\Omega_{M}+4 \Omega_{G} A^{\frac{1}{1+\alpha}} a^{3}}{\Omega_{M}+\Omega_{G} A^{\frac{1}{1+\alpha}} a^{3}} D_{1}^{\prime}-\frac{3}{2} \frac{\Omega_{M}}{\Omega_{M}+\Omega_{G} A^{\frac{1}{1+\alpha}} a^{3}} D_{1}=0,
$$

which is the standard $\Lambda$ CDM equation, Eq. (25), with $\Omega_{\Lambda}=\Omega_{G} A^{\frac{1}{1+\alpha}}$.

The second order equation reads at early times as

$$
D_{2}^{\prime \prime}+\frac{1}{2} D_{2}^{\prime}-\frac{3}{2} \xi D_{2}-\frac{8}{3}\left(D_{1}^{\prime}\right)^{2}-3 \xi D_{1}^{2}=0 .
$$

Substituting the growing mode from Eq. (26), the solution to this equation is

$$
D_{2}=\frac{4}{3} \frac{\sqrt{1+24 \xi}-1-21 \xi}{\sqrt{1+24 \xi}-1-18 \xi} e^{\frac{1}{2}(\sqrt{1+24 \xi}-1) \eta}+B_{3} e^{\frac{1}{4}(\sqrt{1+24 \xi}-1) \eta}+B_{4} e^{-\frac{1}{4}(\sqrt{1+24 \xi}-1) \eta} .
$$

Hence, at early times, the skewness is given by

$$
S_{3}=3 \frac{D_{2}}{D_{1}^{2}}=4 \frac{\sqrt{1+24 \xi}-1-21 \xi}{\sqrt{1+24 \xi}-1-18 \xi}
$$

which when $\Omega_{G}=0$ (i.e. $\xi=1$ ) reduces to the EdS-value, $S_{3}^{E d S}=34 / 7$. At the other extremum point, $\Omega_{M}=0$ or $\xi=0$, we reach $S_{3}=6$. All the other values lie between these two limits. Numerical work (see later section) shows that Eq. (32) is a very good approximation until very late times, but even there the error is very small. 

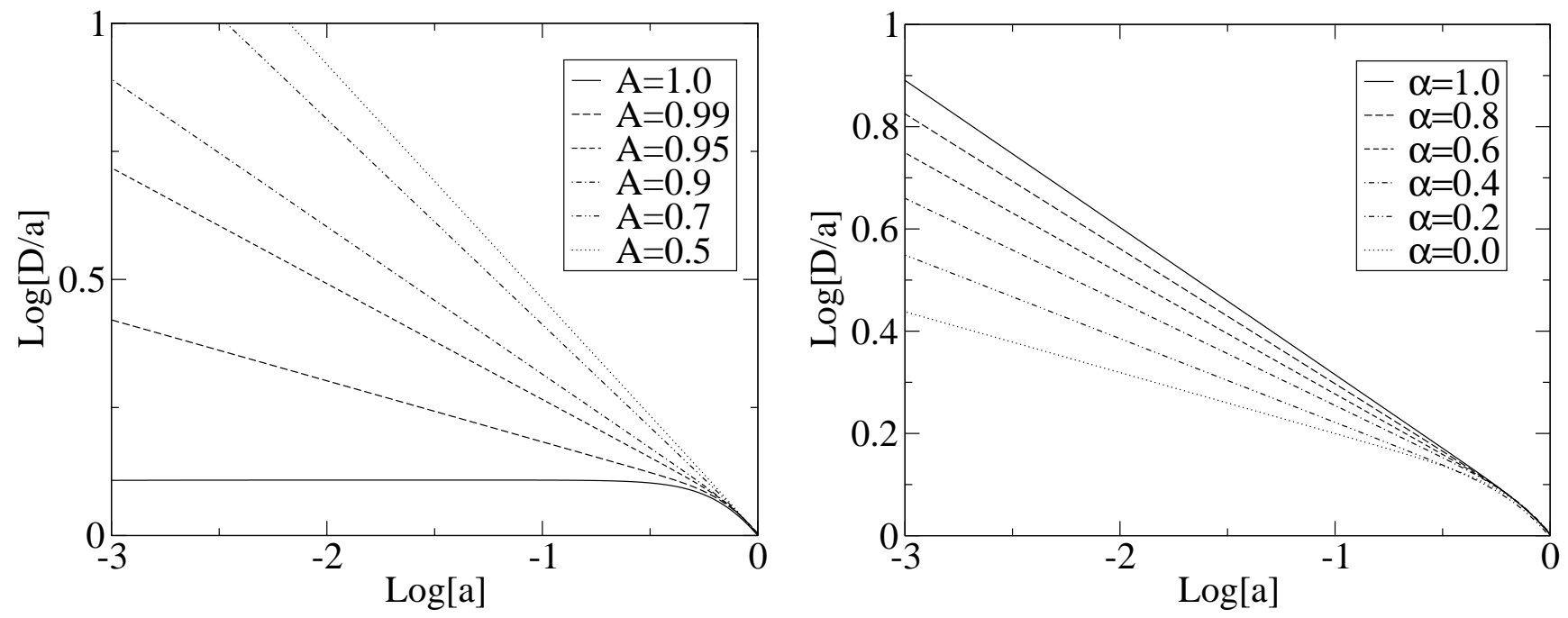

FIG. 2: Linear growth for $\alpha=1.0, A=1,0.95,0.9,0.7,0.5$ (left panel), $A=0.9, \alpha=0,0.2, \ldots, 1.0$ (right panel)

\section{B. Numerical Results}

In order to study the linear and non-linear evolution of the perturbation from early to late times, we must resort to numerical means. The linear and second order equations are solved numerically over the region of cosmological interest, from $a=10^{-3}$ to $a=1 \equiv a_{0}$. The initial conditions are chosen such that in both cases, the growing part of the solution, Eqs (26) and (31), is followed at early times. This ensures that the behavior of the solutions is qualitatively similar to the $\Lambda \mathrm{CDM}$-universe and that the $\Lambda \mathrm{CDM}$-behavior is reached as $A$ tends to unity. Normalization is chosen so that the amplitude of fluctuations is the same at present time, i.e. at $a=1$.

The linear factor is are plotted in Fig 2 Note that the linear growth is depicted relative to $a$, i.e. to the growth rate in the EdS-universe (which is also equal to the growth rate in $\Lambda$ CDM -universe at early times). In plotting the figures, we choose $\Omega_{M}=0.3$, which in a flat universe sets $\Omega_{G}=0.7$.

The linear growth rate confirms what was expected from the analytical results: linear growth occurs much more slowly than in a $\operatorname{EdS}(\Lambda \mathrm{CDM})$ universe until at late times when the $\mathrm{GCg}$ acts as a cosmological constant that dominates, leading to even stronger suppression of growth. Note that the case $A=1.0$ corresponds to the $\Lambda$ CDM universe with $\Omega_{M}=0.3, \Omega_{\Lambda}=0.7$. The effect of changing $\alpha$ is much less significant as is shown in Fig. 2 (right panel).

It is also interesting to study the time derivative of the above ratio: $d F / d r=d(D / a) / d r$, where $d r$ is the comoving distance. This quantity provides a direct observable (see eg [36] and references therein) through the integrated SachsWolfe (ISW) effect [37, 38]. Numerical predictions for $d F / d r$ are shown in Fig. 3. As expected deviations from the standard $\Lambda C D M$ model are substantial for most of the relevant GCg parameters.

The effect on the skewness is not as dramatic and is very well approximated by the analytical formula Eq. (32)

Further details on how these quantities can be compared to observations, can be found in [32, 33] and references therein.

\section{DISCUSSION}

We have considered a simple variation on the Generalized Chaplygin gas cosmology, where in addition to the GCgcomponent there is a significant cold dark matter fraction. The GCg plays the role of dark energy, but not in a unified way (see Introduction) meaning that the GCg does not collapse gravitationally. At first look this model seem viable, as it has quite a standard looking Friedmann equation, see Eq. (2), and cosmological distances, see Fig. 11 However, this is not the case for the growth of structures.

As we have seen both analytically and numerically, the growth of large scale structure is fundamentally different from the $\Lambda \mathrm{CDM}$ scenario due to the properties of the Generalized Chaplygin gas. Unlike in the $\Lambda \mathrm{CDM}-\mathrm{model}$, where at early times the equation governing linear growth equals that of the EdS-case, the GCg behaves differently at early times. From Eq. (28) we see that for $A \neq 1, \rho_{G, 0} \neq 0$, linear growth will be suppressed relative to the $E d S$ scenario, and to a $\Lambda$ CDM universe which follows the EdS-solution until late times. We can see in Fig. 2 that any small deviations of the GCg parameters from the $\Lambda$ CDM values ( $\alpha=1$ and $A=1)$ produced substantial suppression 

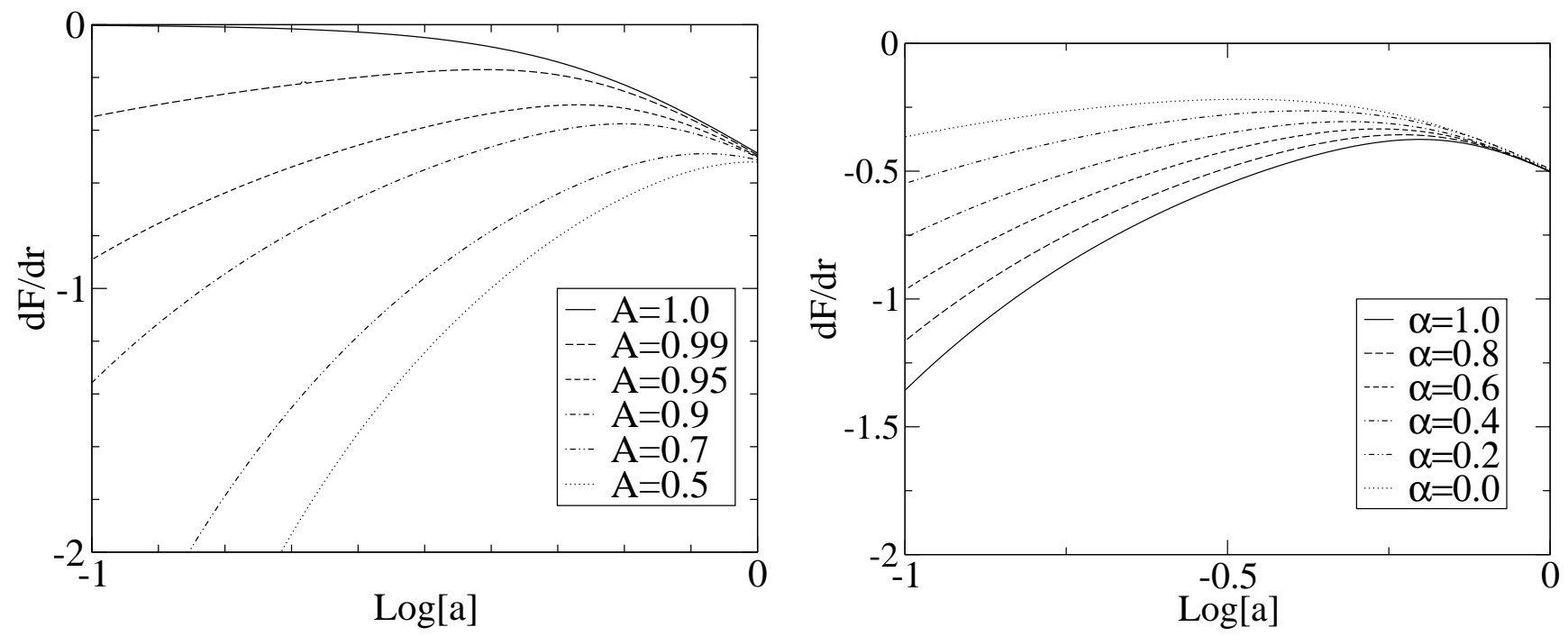

FIG. 3: Derivative of the linear growth: $\dot{F}=d(D / a) / d r$ for $\alpha=1.0, A=1,0.95,0.9,0.7,0.5$ (left panel), $A=0.9, \alpha=$ $0,0.2, \ldots, 1.0$ (right panel)

of linear growth. For example, even the case $A=0.99$ produces a much higher amplitude of fluctuations at decoupling $\left(a \simeq 10^{-3}\right.$ ) than the $\Lambda$ CDM cosmology (when normalized at $a=1$ ). Such differences in normalization are hard to reconcile with our current understanding of CMB anisotropies (see eg [32, 40]). The final numbers dependent on the adopted model for CMB transfer function and matter content, but it is already apparent that we will need to tune the $\mathrm{GCg}$ parameters very close to the $\Lambda \mathrm{CDM}$ model. As an independent test we have also presented the predictions of the GCg model for the ISW effect, see Fig 3 and the skewness of the density field, see Eq.(32). Again, the ISW effect is much larger even for $A=0.99$ compared to a $\Lambda$ CDM universe. It should be stressed that in the scenario considered here, the GCg only acts through the background evolution and does not undergo gravitational collapse. Hence, these parameter constraints do not apply to the unified dark matter models with a GCg component.

Observations seem to favor values of the GCg parameter which makes it equivalent in practice to a cosmological constant. We conclude therefore that using the $\mathrm{GCg}$ as a form of dark energy does not seem to provide any apparent advantage over the more simple $\Lambda$ CDM model, which has fewer parameters. It is nevertheless clear that future observations will be able to provide tighter constraints on the quantities we have studied here. If the $\Lambda$ CDM model fails to match such observations, the GCg models could be an useful alternative to consider.

\section{Acknowledgments}

TM is grateful to the Academy of Finland (grant no. 79447) for financial support and to the Theoretical Physics department of Oxford University for hospitality during the completion of this work. EG and MM acknowledge support from and by grants from IEEC/CSIC and the spanish Ministerio de Ciencia y Tecnologia, project AYA2002-00850 and EC FEDER funding. MM acknowledges support from a PhD grant from Departament d'Universitats, Recerca i Societat de la Informacio de la Generalitat de Catalunya. We are grateful to the Centre Especial de Recerca en Astrofisica, Fisica de Particules i Cosmologia (C.E.R.) de la Universitat de Barcelona and IEEC/CSIC for their support.

[1] P. J. E. Peebles and B. Ratra, Phys. Rev. D37 (1998) 3406; Rev. Mod. Phys. 75 (2003) 599, R. R. Caldwell, R. Dave, P. J. Steinhardt, Phys. Rev. Lett. 8019981582.

[2] G. Dvali, G. Gabadadze and M. Porrati, Phys. Lett. B485 (2000) 208.

[3] K. Freese and M. Lewis, Phys. Lett. B540 (2002) 1.

[4] A. Kamenshchik, U. Moschella and V. Pasquier, Phys. Lett. B511 (2001) 265.

[5] A. Sen, JHEP 0204 (2002) 048.

[6] G. W. Gibbons, Phys. Lett. B537 (2002) 1.

[7] M. Bento, O. Bertolami and A. A. Sen, Phys. Rev. D66 (2002) 043507. 
[8] M. Makler, S. Q. Oliveira and I. Waga, Phys. Lett. B555 (2003) 1.

[9] J. C. Fabris, S. Goncalves and P. de Souza, Gen. Rel. Grav. 34 (2002) 53; Gen. Rel. Grav. 34 (2002) 2111.

[10] N. Bilic, G. Tupper and R. Viollier, Phys. Lett. B535 (2002) 17.

[11] J. C. Fabris, S. Goncalves and R. de Sa Ribeiro, astro-ph/0307028

[12] H. Sandvik et al., astro-ph/0212114

[13] L. M. G. Beca et al., Phys. Rev. D67 (2003) 101301.

[14] R. Bean and O. Dore, astro-ph/0301308

[15] R. Reis et al., astro-ph/0306004

[16] P. Avelino et al., astro-ph/0306493

[17] M. Makler, S. Q. Oliveira and I. Waga, astro-ph/0306507

[18] J. C. Fabris, S. Goncalves and P. de Souza, astro-ph/0207430

[19] R. Colistete et al., astro-ph/0303338

[20] P. Silva and O. Bertolami, astro-ph/0303353

[21] J. Cunha, J. Alcaniz and J. Lima, astro-ph/0306319

[22] P.P. Avelino et al., Phys. Rev. D67 (2003) 023511.

[23] M. Bento, O. Bertolami and A. A. Sen, Phys. Rev. D67 (2003) 063003.

[24] J. Alcaniz, D. Jain and A. Dev, Phys. Rev. D67 (2003) 043514.

[25] M. Bento, O. Bertolami and A. A. Sen, astro-ph/0303538

[26] L. Amendola et al., astro-ph/0304325

[27] D. Carturan and F. Finelli, astro-ph/0211626

[28] A. G. Riess et al., Ap. J. 116 (1998) 1009.

[29] S. Perlmutter et al., Ap. J. 517 (1999) 517.

[30] A. Dev, D. Jain and J. Alcaniz, Phys. Rev. D67 (2003) 023515.

[31] K. Benabed and F. Bernardeau, Phys. Rev. D64 (2001) 083501.

[32] T. Multamäki, E. Gaztañaga and M. Manera, MNRAS 344 (2003), 761, astro-ph/0303526

[33] E. Gaztañaga and J. A. Lobo, Ap. J. 548 (2001) 47.

[34] F. Bernardeau, S. Colombi, E. Gaztañaga, R. Scoccimarro Physíep. 367 (2002) 1.

[35] A. Lue, R. Scoccimarro and G. Starkman, astro-ph/0307034

[36] P. Fosalba, E. Gaztañaga and F. Castander, ApJ in press, astro-ph/0307249

[37] R. K. Sachs and A. M. Wolfe, Ap. J. 147 (1967) 73.

[38] R. Crittenden and N. Turok, Phys Rev. Lett. 76 (1996) 575.

[39] S. Weinberg, Rev. Mod. Phys. 61 (1989) 1.

[40] D. N. Spergel et al., astro-ph/0302209

[41] Sergei A. Chaplygin, 1869-1942 